\title{
Multicast Capacity in Mobile Wireless Ad Hoc Network with Infrastructure Support
}

\author{
Xi Chen, Wentao Huang and Xinbing Wang \\ Department of Electronic Engineering \\ Shanghai Jiao Tong University, China \\ Email: \{qfbzcx,yelohuang,xwang8\}@sjtu.edu.cn
}

\author{
Xiaojun Lin \\ Department of Electrical and Computer Engineering \\ Purdue University, USA \\ Email: linx@ecn.purdue.edu
}

\begin{abstract}
We study the multicast capacity under a network model featuring both node's mobility and infrastructure support. Combinations between mobility and infrastructure, as well as multicast transmission and infrastructure, have already been showed effective ways to increase capacity. In this work, we jointly consider the impact of the above three factors on network capacity. We assume that $m$ static base stations and $n$ mobile users are placed in an ad hoc network, of which the area scales as $f^{2}(n)$. A general mobility model is adopted, such that each user moves within a bounded distance from its home-point with an arbitrary pattern. In addition, each mobile node serves as source of a multicast transmission, which results in a total number of $n$ multicast transmissions. We focus on the situations that base stations actually benefits the capacity, and find that multicast capacity in a mobile hybrid network falls into several regimes. For each regime, matching upper and lower bounds are derived.
\end{abstract}

Index Terms-Wireless ad hoc network; multicast capacity; mobility; infrastructure; hybrid network; scaling law;

\section{INTRODUCTION}

Recent years witness a rapid development in wireless ad hoc network, in both academic and industrial fields. Kumar and Gupta has showed in their ground breaking work [1] that, even with the optimal scheduling, routing and relaying of packets, the per-node capacity still decreases as $\Theta(1 / \sqrt{n})$ when $\mathrm{n}$ tends to infinity. Lots of works try to improve this disappointing scalability of throughput capacity by introducing different characteristics into ad hoc network, such as mobility of nodes, an infrastructure of the network, a multicast transmission scheme, etc.

Mobility in ad hoc network has been considered firstly by D.Tse et al. in [2]. A store-carry-forward relaying scheme was proposed and proven to sustain a $\Theta(1)$ per-node capacity, if each node can visit the whole network area with an uniformly ergodic mobility process. M. Garetto et al. generalize the mobility through a restriction that each moving node is located within a circle of radius $1 / f(n)$ [3]. By mapping the network to a generalized random geometric graph, the authors have proven that a $\Theta(1 / f(n))$ per-node capacity is achievable.

Infrastructure in ad hoc network provides a more straightforward increase to the capacity. In [4], B. Liu et al. claim that infrastructure can offer a linear capacity increase in hybrid network, when the number of base stations increases asymptotically faster than $\sqrt{n}$. In addition, Kozat and Tassiulas [5] prove that if the number of users served by each BS is bounded above, a per-node capacity of $\Theta(1 / \log n)$ can be achieved. In [6], Agarwal and Kumar further extend this result to $\Theta(1)$.

Multicast transmission refers to the transmission from single node to other $k-1$ nodes, so as to generalize both unicast and broadcast transmissions. In [7], X.-Y. Li proves that multicast transmission can obtain a per-flow capacity of $\Theta\left(\sqrt{\frac{1}{n \log n}}\right.$. $\left.\frac{1}{\sqrt{k}}\right)$, which is larger than that of $k$ unicast transmissions. The gain of multicast transmission results from a merge of relay paths within a minimum spanning tree. In [8], X-Y. Li et al. extend the multicast transmission to Gaussian channel model and show similar capacity improvement under corresponding protocol.

Many succeeding literatures focus on the combinations of the above characteristics. Some aim to further increase the network performance, while others try to present a more realistic scenario. In [9] and [10], X-Y. Li et al. explore the multicast capacity in a static hybrid network with infrastructure support. Establishing the multicast tree with the help of infrastructure and employing a hybrid routing, the authors have showed that the achievable multicast capacity in hybrid network with $m$ BSs is $\Theta\left(\max \left[\min \left(\frac{W_{B} \sqrt{m}}{n_{s} \sqrt{k}}, \frac{W_{c} \sqrt{m}}{n_{s} k}, \frac{W_{a} \sqrt{m}}{n_{s} k}\right), \frac{W_{a}}{n_{s} \sqrt{k}} \cdot \frac{a}{r}\right]\right)$. On the other hand, W. Huang, X. Wang and et al. study the unicast capacity of mobile hybrid network in [11], which jointly consider the influences of node's mobility and infrastructure. A per-node capacity is $\Theta\left(\frac{1}{f(n)}\right)+\Theta\left(\min \left[\frac{m^{2} c}{n}, \frac{m}{n}\right]\right)$ for strong mobility, and $\Theta\left(\min \left[\frac{m^{2} c}{n}, \frac{m}{n}\right]\right)$ for weak and trivial mobility.

In this paper, we further study the multicast capacity scaling laws of a mobile hybrid network characterizing both mobility and infrastructure. In our model, each of the $n$ users moves around a home-point within a bounded radius. $m$ wire-connected base stations are placed in a wireless ad hoc network, of which the area scales with $\mathrm{n}$ as $f^{2}(n)$. A multicast path can be generated with infrastructure routing and pure ad hoc routing, as well as a combination of both.

Our main contributions: In this paper, we consider the roles of mobility and infrastructure in multicast capacity of a wireless mobile ad hoc network. We divide mobility into three regimes, and present matching upper bounds and lower bounds for each regime. We assume that bandwidth is $W$ for 
wireless channel, and $W_{B}$ for wired connections. In cellular routing, we further divide wireless frequency resource $W$ into uplink bandwidth $W_{A}$ and downlink bandwidth $W_{C}$.

- For the first regime (strong mobility regime) where $f(n) \sqrt{\gamma(n)}=o(1),\left(\gamma(n)=\frac{\log n_{c}}{n_{c}}\right)$, the per-node capacity by ad hoc routing is:

$$
\Theta\left(\frac{W}{\sqrt{k} f(n)}\right)
$$

And per-node capacity by cellular routing is:

$$
\Theta\left(\min \left[\frac{m W_{A}}{n}, \frac{m^{2} W_{B}}{k n}, \frac{m W_{C}}{k n}\right]\right)
$$

By always choosing a better routing, the per-node capacity of hybrid routing is:

$$
\Theta\left\{\max \left[\frac{W}{\sqrt{k} f(n)}, \min \left(\frac{m W_{A}}{n}, \frac{m^{2} W_{B}}{k n}, \frac{m W_{C}}{k n}\right)\right]\right\}
$$

- The second regime (weak mobility regime) stands for the situation that $f(n) \sqrt{\gamma(n)}=\omega(1)$ and $f(n) \sqrt{\tilde{\gamma}(n)}=$ $o(1)$, where $\sqrt{\tilde{\gamma}(n)}=\frac{1}{r} \sqrt{\frac{\log \left(n / n_{c}\right)}{n / n_{c}}}$. We prove that, in this regime, mobility is only helpful in delivering intra-cluster message. The inter-cluster message can only be transmitted through cellular routing. As a result, the optimal routing scheme is a serial connection of ad hoc routing (phase 1) and cellular routing (phase 2). We prove that the result in the first regime can be mapped to phase 1 here. Consequently, the per-node capacity by ad hoc routing is:

$$
\Theta\left(\frac{W \sqrt{n_{c}}}{n^{R} \sqrt{k}}\right)
$$

Furthermore, the per-node capacity by cellular routing is:

$$
\Theta\left[\min \left(\frac{m W_{A}}{n}, \frac{n_{c} m^{2} W_{B}}{\left(n_{c}-1\right) n k}, \frac{m n_{c} W_{C}}{n k}\right)\right]
$$

Considering the nature of serial connection, the per-node capacity by hybrid routing is:

$$
\Theta\left[\min \left(\frac{W \sqrt{n_{c}}}{n^{R} \sqrt{k}}, \frac{m W_{A}}{n}, \frac{n_{c} m^{2} W_{B}}{\left(n_{c}-1\right) n k}, \frac{m n_{c} W_{C}}{n k}\right)\right]
$$

- The third regime (trivial mobility regime) corresponds to $f(n) \sqrt{\tilde{\gamma}(n)}=\omega(1)$. In this regime, we can prove that mobility is trivial and the network acts as a static one. Previous conclusions in static hybrid network, such as [4], [9], and [10], can be applied in this trivial mobility regime.

Different from previous works on hybrid network [4][6], [9], [10], our work takes node's mobility into account. Besides, our work is the first one to consider the effect of a general mobility on multicast transmission. Furthermore, we study multicast capacity in a more realistic network model featuring both mobility and infrastructure. As a result, our work generalizes both unicast and broadcast capacity results on MANETs and hybrid network.

This paper is organized as follows. We first introduce our network models and assumptions in Section II. Section III and
Section IV define the uniformly dense networks, and derive multicast capacity in such network under ad hoc routing and cellular routing, respectively. In Section V, we combine the results of previous two sections and derive multicast capacity under hybrid routing. Capacity analysis of non-uniformly dense networks is carried out in Section VI. At last, we conclude our work in Section VII.

\section{Models And Assumptions}

We consider a wireless network consisted of $n$ mobile users(also referred as mobile stations, MSs) moving over a bidimensional surface. Communications are carried out in wireless channel with bandwidth $W$ by ad hoc routing, or by cellular routing with the help of $m=\Theta\left(n^{M}\right)$ base stations (BSs), which are connected to each other by optical fibers with bandwidth $W_{B}$.

We use $X_{i}(t)$ to denote the position of $i$ th MS at any given time $t$, and $Y_{i}(t)$ for $i$ th BS. Since BSs are statically placed in the network, we have $\forall t, Y_{i}(t) \equiv Y_{i}(0) \triangleq Y_{i}$. When referring to both MSs and BSs, we adopt $Z_{i}(t), 1 \leq i \leq n+m$ to denote their locations. $X_{i}^{h}$ is used to denote the location of home-point for $i$ th MS. We further define operator $\|\cdot\|$ as the distance between two points: $d_{i j}=\left\|Z_{i}-Z_{j}\right\|$.

\section{A. Mobility Model}

Definition 1: (Network Extension) $\mathcal{O}$ is a Torus, or a square with wrap-around conditions. The side length of the network scales with $n$ according to a non decreasing function $f(n)=$ $n^{\alpha}$, where $\alpha \in[0,1 / 2]$. We normalize the whole network to a unit Torus for convenience. Correspondingly, any value representing a distance in the network should be scaled down by $1 / f(n)$.

Remark 1: $\alpha=0$ corresponds to the dense network [1], of which the size remains constant while node density increases linearly with $n$. And $\alpha=1 / 2$ represents the extended network [12], in which network size increase linearly with $n$ while node density remains constant.

It is worth pointing out that ignoring the edge effect of a network is reasonable for avoiding tedious derivations, and is adopted in previous works [3], [11]. At the same time, normalization of the network area is commonly used for convenience [1], [2]. The above assumptions will not influence the main results in this paper.

Definition 2: (Home-Point Cluster Model) We assume there are $n_{c}=\Theta\left(n^{C}\right)$ clusters with radius $r=\Theta\left(n^{-R}\right)$. All the clusters are independently and uniformly distributed in network $\mathcal{O}$. Then each of the $n$ home-points is randomly assigned to a cluster and placed in it uniformly and independently.

Definition 3: (MS Mobility) We assume that $\left\{X_{i}(t)\right\}$ are independent, stationary, ergodic and rotation-invariant processes with stationary distribution $\phi_{i}(X)$ :

$$
\phi_{i}(X)=\phi_{i}\left(X-X_{i}^{h}\right)=\frac{s\left(\left\|X-X_{i}^{h}\right\|\right)}{\int_{\mathcal{O}} s\left(\left\|X-X_{i}^{h}\right\|\right) d X}
$$


,where $s(d)$ is an arbitrary non-decreasing function with finite range.

Remark 2: $s(d)$ describes MS's presence around its homepoint before network normalization. Since

$$
\int_{\mathcal{O}} s\left(\left\|X-X_{i}^{h}\right\|\right) d X \sim 1 / f^{2}(n)
$$

we have:

$$
\phi_{i}(X) \sim f^{2}(n) s\left(\left\|X-X_{i}^{h}\right\|\right)
$$

Remark 3: Our mobility model is similar to that of [3] and [11]. In order to characterize the non-uniform distribution and limited motion observed in the real mobility(see [13], etc.), home-points and $s(d)$ are introduced in our work. Such model can better demonstrate the preferential attachment phenomena in real networks. Furthermore, our mobility and cluster model can generalize other classical mobility models, such as i.i.d. mobility models [12], hybrid random walk models [14] and Brownian motion models [15], by setting $n^{C}=n$ and $f(n)=$ $\Theta(1)$.

Definition 4: (Mobility Regimes) Let $\gamma(n)=\frac{\log n_{c}}{n_{c}}$ and $\tilde{\gamma}(n)=\frac{1}{r^{2}} \log \frac{\left(n / n_{c}\right)}{n / n_{c}}$. We say that MS's mobility is strong if $f(n) \sqrt{\gamma(n)}=o(1)$. Weak mobility corresponds to $f(n) \sqrt{\gamma(n)}=\omega(1)$ and $f(n) \sqrt{\tilde{\gamma}(n)}=o(1)$, while trivial mobility corresponds to $f(n) \sqrt{\tilde{\gamma}(n)}=\omega(1)$

Remark 4: With strong mobility, MSs can exist cluster boundaries and meet those in other clusters. Under weak mobility, MSs only move within their own clusters, and mobility only helps in delivering intra-cluster message. In the case of trivial mobility, mobility provides little help in forwarding packets, and the whole network acts like a static one. Also, the mobility regime is an attribute of network, rather than that of MSs. The determinants of mobility regimes are growing speed of network size and clustering level of homepoints.

Instead of regularly BS placing method in [4], [9] and [10], the distribution of BSs in this paper matches that of MSs, so as to achieve better utilization of BSs. For $j$ th BS, we randomly select a point $Q_{j}$ according to the cluster model, then uniformly and independently placed the BS in this cluster with distribution $\phi\left(Y-Q_{j}\right)$.

In this paper, we focus on the case that clusters do not overlap with each other w.h.p., which is guaranteed by $M<$ $2 R$. Also, we assume $0 \leq R \leq \alpha$, so that clusters will not shrink with increasing $n$. In order to ensure that every cluster is served by BSs w.h.p., i.e. $m=\omega\left(n_{c}\right)$. An example network under cluster and mobility models is showed in Fig. 1.

\section{B. Communication and Interference Models}

Base stations communicate with each other through optical fiber with bandwidth $W_{B}$. This kind of communication will not cause interference to itself or wireless communications. We assume that available bandwidth in all the wireless channels is $W$. In ad hoc routing, transmissions fully occupy the wireless bandwidth $W$. In cellular routing, bandwidth is further divided into uplink bandwidth $W_{A}$ and downlink bandwidth $W_{C}$. All

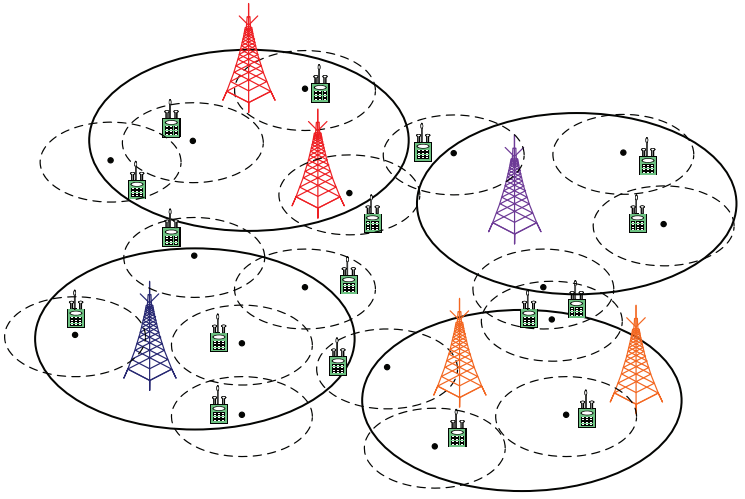

Fig. 1. An example network under cluster and mobility models. We use solid lines for cluster boundaries, dash lines for mobility boundaries, and solid points for home-points.

the communications in wireless channels are characterized by Protocol Model, which is defined as followed.

Definition 5: (Protocol Model) Both BSs and MSs adopt the same transmission range $R_{T}$ (correspondingly, same transmission power). At each time slot $t$, a wireless transmission from node $i$ to node $j$ is successful only if: 1)

$$
\left\|Z_{i}(t)-Z_{j}(t)\right\| \leq R_{T}
$$

and 2)For any other node $l$ that transmits at $t$,

$$
\left\|Z_{l}(t)-Z_{j}(t)\right\| \geq(1+\Delta) R_{T}
$$

, where $\Delta$ is a constant defining protection zone.

We assume all of the $n$ MSs are source nodes, each of which randomly selects other $k \mathrm{MSs}$ as destinations and transmits the same data to them. The selection of destinations is welldesigned, such that every MS is the destination of other $k$ MSs. Correspondingly, We define the Feasible Throughput and Asymptotic Multicast Per-node Capacity $\lambda$ similar to previous works, such as [1], [7], etc..

\section{Multicast Capacity in Uniformly Dense NeTwORK BY AD Hoc Routing}

In this section, we firstly provide a definition of uniformly dense network, as well as some characteristics in such network. We show that when a network falls into strong mobility regime, it is equivalent to classify it as an uniformly dense network. Then matching upper and lower bounds are presented in both pure ad hoc routing and cellular routing for uniformly dense network. For pure ad hoc routing, we map the mobile network into a random geometric graph, and derive matching capacity bounds. And it is worth pointing out that our result is the first one to analysis the multicast capacity under general mobility model. For cellular routing, we divide the routing scheme into three phase and achieve matching upper and lower bounds in each phase, as well. 


\section{A. Preliminary for Uniformly Dense Network}

Definition 6: (Local Density) The local density of nodes at any given point $X_{0}$ is defined as:

$$
\rho^{n}\left(X_{0}\right)=\sum_{i=1}^{n} E\left[\mathbf{1}_{X_{i}^{(n)} \in B\left(X_{0}, 1 / \sqrt{n}\right)} \mid \mathbf{H}_{\mathbf{n}}\right]
$$

,where $B\left(X_{0}, 1 / \sqrt{n}\right)$ is the disk centering at $X_{0}$ with radius $1 / \sqrt{n} . \mathbf{H}_{\mathbf{n}}=\left\{Z_{i}^{h}\right\}_{i=1}^{n+m}$ defines the Borel-field of homepoints. $E$ stands for expectation, and $\mathbf{1}_{x}$ represents the indicator function.

Definition 7: (Uniformly Dense Network) We say a network is uniformly dense if for any $X \in \mathcal{O}$, there exist two positive constant $q$ and $Q$, such that

$$
q<\rho^{n}\left(X_{0}\right)<Q
$$

Lemma 1: Suppose that $Z_{i}^{h}$ are placed on $\mathcal{O}$ according to cluster model. Let $\mathcal{A}$ be any given regular tessellation of $\mathcal{O}$, with area $|\mathcal{A}| \geq(16+\delta) \gamma(n)$, for some small $\delta>0$. Let $N(\mathcal{A})$ be the number of home-points of both BSs and MSs inside $\mathcal{A}$. Then it holds w.h.p. :

$$
\frac{n|\mathcal{A}|}{2} \leq \inf N(\mathcal{A}) \leq \sup N(\mathcal{A}) \leq 2 n|\mathcal{A}| .
$$

Lemma 2: If $f(n) \sqrt{\gamma(n)}=o(1)$ and $m=O(n)$, where $\gamma(n)=\frac{\log \left(n_{c}\right)}{n_{c}}$. then the network is uniformly dense.

The proof of Lemma 1 and Lemma 2 are available in both [11] and [16].

Remark 5: $f(n) \sqrt{\gamma(n)}=o(1)$ indicates that nodes can move outside their the home-point clusters, and may move into other clusters. In this sense, mobility plays a fundamental role in exchanging information. Otherwise, nodes cannot exit their cluster and communicate with those in other clusters. Therefore, clustering becomes a obstacle of connectivity.

Definition 8: (Link Capacity) Link capacity between node $i$ and $j$ is defined by the maximal long term data flow between them:

$$
\mu^{S}(i, j)=E\left[\mathbf{1}_{(i, j) \in \pi^{S}(t)} \mid \mathbf{H}_{n}\right]
$$

,where $S$ is any given scheduling under protocol model, and $\pi^{S}(t)$ denotes the set of transmission pairs scheduled by $S$.

\section{B. Upper Bound in Uniformly Dense Network by Ad Hoc Routing}

By ad hoc routing, we means that MSs only exchange information in wireless channel with a bandwidth $W$, ignoring the effect of BSs.

Definition 9: (Scheduling Scheme $S^{*}$ ) $S^{*}$ schedules node $i$ transmit to node $j$ at time slot $t$ only if the following two condition are both satisfied: 1) $\left\|Z_{i}(t)-Z_{j}(t)\right\|<R_{T}=\frac{c_{T}}{\sqrt{n}}$, and 2) For any other node $l$, whether active or silent, $\| Z_{l}(t)-$ $Z_{j}(t) \|>(1+\Delta) R_{T}$.

The next lemma is provided by [3]. It establishes a relationship between link capacity and encounter probability considering mobility.
Lemma 3: In uniformly dense network with bandwidth $W$, under scheduling $S^{*}$, the link capacity between two nodes(at least contain one MS) is:

$$
\mu^{S^{*}}(i, j)=\Theta\left(W \cdot \operatorname{Pr}\left\{d_{i j} \leq \frac{c_{T}}{\sqrt{n}} \mid \mathbf{H}_{\mathbf{n}}\right\}\right)
$$

Corollary 1: The link capacities between MSs $i, j$ and BS $l$ are:

$$
\begin{aligned}
\mu\left(X_{i}^{h}, X_{j}^{h}\right) & =\Theta\left(W \cdot \frac{f^{2}(n)}{n} \eta\left(f(n)\left\|X_{i}^{h}-X_{j}^{h}\right\|\right)\right) \\
\mu\left(X_{i}^{h}, Y_{l}^{h}\right) & =\Theta\left(W \cdot \frac{f^{2}(n)}{n} s\left(f(n)\left\|Y_{l}^{h}-X_{i}^{h}\right\|\right)\right)
\end{aligned}
$$

Proof: The proof of equation (4) is reported in [3], we only present the proof of equation (5) here.

$$
\begin{aligned}
\mu\left(X_{i}^{h}, Y_{l}^{h}\right) \sim W \cdot f^{2}(n) \int_{Y_{l} \in \mathcal{O}} \int_{X_{i} \in B\left(Y_{l}, R_{T}\right)} \\
\\
=W \cdot s\left(f(n)\left\|X_{i}-X_{i}^{h}\right\|\right) \delta\left(Y_{l}-Y_{l}^{h}\right) d X_{i} d Y_{l} \\
=W \cdot f^{2}(n) \int_{X_{i} \in B\left(Y_{l}, R_{T}\right)} s\left(f(n)\left\|X_{i}-X_{i}^{h}\right\|\right) d X_{i} \\
\sim W \cdot f^{2}(n)\left|B\left(Y_{l}, R_{T}\right)\right| s\left(f(n)\left\|Y_{l}^{h}-X_{i}^{h}\right\|\right) \\
=W \cdot \frac{\pi c_{T}^{2} f^{2}(n)}{n} s\left(f(n)\left\|Y_{l}^{h}-X_{i}^{h}\right\|\right)
\end{aligned}
$$

where $\delta(\cdot)$ denotes the Dirac impulse.

It is easy to prove that scheduling $S^{*}$ is optimal in order sense. On one hand, $\forall R_{T}=o(1 / \sqrt{n})$ can not fully guarantee connectivity and results in a performance decline. On the other, $\forall R_{T}=\omega(1 / \sqrt{n})$ will cause too much interference and decreases the link capacity, as well. ${ }^{1}$

By mapping the network into a Generalized Random Geometric Graph $G\left(n, n_{c}, r, \mu\right)$, we can calculate the asymptotic capacity more conveniently. In $G\left(n, n_{c}, r, \mu\right)$, we use $n$ vertices to represent the home-points. For each pair of vertices $(i, j)$, we connect them with an edge of capacity $\mu\left(\left\|X_{i}^{h}-X_{j}^{h}\right\|\right)$. The following proposition provides an upper bound of multicast capacity in the network.

Proposition 1: Define that $d_{i D}^{h}$ is the length of minimum spanning tree that covers source node $i$ and the corresponding home-points of its $k$ destinations. If throughput $\lambda$ is sustainable, we have:

$$
\lambda \sum_{s} d_{s D}^{h} \leq \sum_{i j} \mu_{i j}^{h} d_{i j}^{h}
$$

Proof: For any source-destination pair $(s, d)$, traffic $\lambda$ must pass through relay pathes connecting $(s, d)$ in graph $G\left(n, n_{c}, r, \mu\right)$. By the definition of minimum spanning tree and triangle inequality, all the relay pathes connecting source $s$ and destinations $D=\left\{d_{1}, d_{2}, \ldots d_{k}\right\}$ should have a total length larger than $d_{s D}^{h}$.

Fortunately, we have a classic conclusion on $d_{s D}^{h}$.

Lemma 4: In a d-dimensional cube of side length $a$, Euclidian minimum spanning tree of $n$ randomly and uniformly

\footnotetext{
${ }^{1}$ A formal proof for the optimality of scheduling $S^{*}$ is presented in [11].
} 
distributed nodes has an asymptotic length of $\tau(d) n^{\frac{d-1}{d}}$, where $\tau(d)$ only depends on $d$.

Corollary 2: $\forall i, 1 \leq i \leq n, d_{i D}^{h}=\Theta(\sqrt{k})$.

Theorem 1: The upper bound of per-node multicast capacity in uniformly dense network by ad hoc routing is:

$$
\lambda=O\left(\frac{W}{\sqrt{k} f(n)}\right)
$$

Proof: In the first step, we try to simplify equation (6) and derive an preliminary expression of upper bound using Proposition 1. We introduce a sum: $T^{n}(d)=-\sum_{i} \sum_{j} \mu_{i j}^{n} \mathbf{1}_{d_{i j}^{h}>d}$, which represents the opposite summation of link capacities provided by links satisfying $d_{i j}^{h}>d$. By definition, it is obvious that $T(d+\Delta)-T(d)=\sum_{i} \sum_{j} \mu_{i j}^{n} \mathbf{1}_{d<d_{i j}^{h}<d+\Delta}$, so we have :

$$
\sum_{i} \sum_{j} \mu_{i j}^{n} d_{i j}^{h}=\int x d T^{n}(x)
$$

Applying this to Proposition 1 results in:

$$
\lambda \sum_{s} d_{s D}^{h} \leq x d T^{n}(x)
$$

Combining with Corollary 2, we have:

$$
\lambda=O\left(\frac{1}{n \sqrt{k}} \int x d T^{n}(x)\right)
$$

In the second step, we calculate $\int x d T^{n}(x)$ to finish the proof. Let $D=\Theta(1 / f(n))$, we can divide $\int x d T^{n}(x)$ into $\int_{x \leq D} x d T^{n}(x)$ and $\int_{x>D} x d T^{n}(x)$.

On one hand, $\int_{x \leq D} x d T^{n}(x)$ is summation of link capacities whose home-points are within distance $\Theta(1 / f(n))$. This part of integration sums up link capacities only when two nodes are close to each other. In other words, there exists a constant $y_{0}$, such that $\operatorname{Pr}\left\{d_{i j} \leq R_{T}(n)\right\}>0$ only when $\left\|X_{i}^{h}-X_{j}^{h}-\right\| \leq \frac{y_{0}}{f(n)}$. Meanwhile, considering the limited bandwidth for each node, it is obvious that $\mu_{i}^{n}=\sum_{j} \mu_{i j}^{n} \leq W$. Assume that there is a simple curve $\mathcal{L}$ dividing $\mathcal{O}$ into $A_{0}$ and $B_{0}$. The information flow crossing $\mathcal{L}$ is $\sum_{i \in A_{0}} \sum_{j \in B_{0}} \mu_{i j}^{n}$. Define $C_{0}$ as the set of nodes that are within $\frac{y_{0}}{f(n)}$ away of $\mathcal{L}$. Combining with premise that $x \leq D$, we have:

$$
\begin{aligned}
\sum_{i \in A_{0}} \sum_{j \in B_{0}} \mu_{i j}^{n} & =\sum_{i \in C_{0}} \sum_{j \in B_{0}} \mu_{i j}^{n} \\
& \leq \sum_{i \in C_{0}} \mu_{i}^{n} \leq W \cdot N\left(C_{0}\right) \leq \Theta\left(\frac{W \cdot n}{f(n)}\right)
\end{aligned}
$$

Substituting this into (8), it follows:

$$
\lambda=O\left(\frac{W}{\sqrt{k} f(n)}\right)+O\left(\frac{1}{n \sqrt{k}} \int_{x>\frac{1}{f(n)}} x d T_{n}(x)\right)
$$

On the other hand, we try to find a simple lower bound $S^{n}(d)$ of $T^{n}(d)$ to calculate $\int_{x>D} x d T^{n}(x)$. The $S^{n}(d)$ should satisfy 1) $\sup ^{n}(d)=0$ and 2) $T^{n}(d) \geq S^{n}(d)$. And this results in:

$$
\int_{x>D} x d T^{n}(x) \leq \int_{x>D} x d S^{n}(x)
$$

By definition of transmission range, we have:

$$
\begin{aligned}
\operatorname{Pr}\left\{d_{i j}(t) \leq R_{T}\right\} \leq & \operatorname{Pr}\left\{\left\|X_{i}(t)-X_{i}^{h}>\frac{d_{i j}^{h}-R_{T}}{2}\right\|\right\} \\
& +\operatorname{Pr}\left\{\left\|X_{j}(t)-X_{j}^{h}>\frac{d_{i j}^{h}-R_{T}}{2}\right\|\right\} \\
= & 2 \int_{\mathcal{O}} \phi^{n}(x) \mathbf{1}_{x>\frac{d_{i j}^{h}-R_{T}}{2}} d x
\end{aligned}
$$

Considering definition of $T(d)$, we have:

$$
\begin{aligned}
T(d) & \geq W \cdot-\sum_{i} E\left[\mathbf{1}_{\left.\left\|X_{i}(t)-X_{i}^{h}\right\|>\frac{d-R_{T}}{2}\right]}\right. \\
& \geq-2 n W \cdot \int_{\mathcal{O}} \phi^{n}(x) \mathbf{1}_{x>\frac{d_{i j}^{h}-R_{T}}{2}} d x=S(d)
\end{aligned}
$$

For any constant $M$, it follows:

$$
\begin{aligned}
& \int_{x>D} x d T^{n}(x) \\
& \leq \frac{-W}{n \sqrt{k}} \int_{y>\frac{M}{f(n)}} 2 n y \cdot \frac{d}{d y}\left(\int_{X \in \mathcal{O}} \phi^{n}(\|X\|) \xi(\|X\|) d X\right) d y
\end{aligned}
$$

where $\xi(\|X\|)=\mathbf{1}_{\|X\|>\frac{d_{i j}^{h}-R_{T}}{2}}$ We take $M=10$ (which does not affect the result in order sense), and when $n$ is large enough, for any $y \geq \frac{10}{f(n)}$, it is true that $\frac{y-R_{T}}{2}>\frac{y}{3}$. So that:

$$
\begin{aligned}
& \int_{x>D} x d T^{n}(x) \\
& \leq \frac{-W}{n \sqrt{k}} \int_{y>\frac{10}{f(n)}} 2 n y \cdot \frac{d}{d y}\left(\int_{X \in \mathcal{O}} \phi^{n}(\|X\|) \xi(\|X\|) d X\right) d y \\
& \approx \frac{2 W}{\sqrt{k}} \int_{y>\frac{10}{f(n)}} y\left[2 \pi y \phi^{n}\left(\frac{y}{3}\right)\right] d y \\
& <\frac{4 \pi W}{\sqrt{k}} \int_{y} f^{2}(n) y^{2} \phi\left(f(n)\left(\frac{y}{3}\right)\right) d y=\Theta\left(\frac{W}{\sqrt{k} f(n)}\right)
\end{aligned}
$$

Finally, we have:

$$
\lambda=O\left(\frac{W}{\sqrt{k} f(n)}\right)+O\left(\frac{W}{\sqrt{k} f(n)}\right)=O\left(\frac{W}{\sqrt{k} f(n)}\right)
$$

\section{Lower Bound in Uniformly Dense Network by Ad Hoc Routing}

In the following part of this section, we will derive asymptotically matching lower bound on multicast capacity in uniformly dense network by ad hoc routing. We have already mapped a mobile network into static graph, which makes the establishment of a multicast routing possible and realistic. We employ the next algorithm from [7] to set up the multicast routing in graph $G\left(n, n_{c}, r, \mu\right)$. 
Algorithm 1 Optimal ad hoc routing for multicast transmission $U_{i}=\left\{s_{i}, d_{i, 1}, d_{i, 2}, \ldots, d_{i, k}\right\}$

1. Divide $\mathcal{O}$ into squarelets, of which the side length is $c / f(n)$. And we will have $f(n) / c$ such squarelets. We denotes the squarelet in row $i$ column $j$ as $(i, j)$.

2. We use the following scheme to generate the Euclidian Spanning Tree $\operatorname{EST}\left(U_{i}\right)$

(1) Let the $k+1$ nodes form $k+1$ connected components;

(2) repeat (3) and (4) for $g=1,2,3, \ldots, k$;

(3) in the $g$ th step, divide $\mathcal{O}$ into at most $k-g$ square cells, each with side length $\frac{1}{\sqrt{k-g}}$;

(4) choose a cell that contain two nodes from different connected components, then connect them using Manhattan routing and merge the corresponding components.

3. For each link $u v$ in $\operatorname{EST}\left(U_{i}\right)$, assume that $u$ and $v$ are located inside squarelet $\left(i_{u}, j_{u}\right)$ and $i_{v}, j_{v}$. In squarelet $\left(i_{u}, j_{v}\right)$ or $\left(i_{v}, j_{u}\right)$, choose a node $w$, so that $u w v$ is the Manhattan routing connecting $u$ and $v$. Repeat this for each link in $\operatorname{EST}\left(U_{i}\right)$, and merge the corresponding link into Manhattan tree $M T\left(U_{i}\right)$.

4. For each link $u w$, find a node in each of the squarelets that are crossed by line $u w$. Obtain a path $P(u, w)$ by connecting all of this nodes. Then delete those not in $U_{i}$ and obtain the multicast tree $\operatorname{MTR}\left(U_{i}\right)$.

5. Return $M T R\left(U_{i}\right)$.

Lemma 5: By Algorithm III-C, the probability that a random multicast flow will be routed through a certain squarelet $A$ is at most $c^{\prime} \sqrt{k} c / f(n)$.

Proof: Assume that $A$ is located in row $i$ column $j$ of a $L \times L$ grid network. We randomly pick two nodes $u$ and $v$ , and connect them with Manhattan routing.Let $\mathbf{I}_{A}$ denote the event that this routing crosses $A$, and $p_{s}(L)=\operatorname{Pr}\left\{\mathbf{I}_{A}=1\right\}$. By the characteristic of Manhattan routing, we have:

$$
p_{s}(L)=\frac{i-1}{L^{2}} \cdot \frac{L-i+1}{L}+\frac{j-1}{L^{2}} \cdot \frac{L-j+1}{L}
$$

,which results: $\frac{2}{L^{2}} \leq p_{s}(L) \leq \frac{2}{L}$. Let $\mathbf{I}_{A, g}$ denote the event that $A$ is used in the $g$ th step of Algorithm III-C, it follows:

$$
\operatorname{Pr}\left\{\mathbf{I}_{A, g}\right\}=\frac{1}{k-g} \cdot p_{s}\left(\frac{1 /(\sqrt{(k-g)})}{c / f(n)}\right)
$$

In all, we have:

$$
\begin{aligned}
p & \leq \sum_{g=1}^{k-1} \operatorname{Pr}\left\{\mathbf{I}_{A, g}\right\} \\
& =\sum_{g=1}^{k-1} \frac{1}{k-g} \cdot p_{s}\left(\frac{1 /(\sqrt{(k-g)})}{c / f(n)}\right) \leq \frac{4 \sqrt{2}}{5} \sqrt{k} \frac{c}{f(n)}
\end{aligned}
$$

Setting $c^{\prime}=\frac{4 \sqrt{2}}{5}$ finishes the proof.

Theorem 2: With the MTR generated by Algorithm III-C, the sustainable per-node multicast capacity by ad hoc routing in dense network is $\lambda=\Theta\left(\frac{1}{\sqrt{k} f(n)}\right)$.
Proof: In a random geometric graph $G$, number of edges from squarelet $A$ to squarelet $B$ is $N(A) N(B)$. If a per-node capacity $\lambda$ is sustainable, each of these edges should not be overloaded. Considering the maximum of flows crossing one node defined by Lemma 5 , we have:

$$
\begin{aligned}
\frac{\lambda \frac{c^{\prime} c n \sqrt{k}}{f(n)}}{\underline{N}(A) \underline{N}(B)} & =\Theta\left(\lambda \cdot \frac{\sqrt{k} f^{3}(n)}{n}\right) \\
& \leq W \cdot \mu^{n}\left(\bar{d}_{A, B}\right)=W \cdot g(n) \eta(\sqrt{5} c)
\end{aligned}
$$

where $\bar{d}_{A, B}=\frac{\sqrt{5} c}{f^{3}(n) \sqrt{k} / n}$. To sum up, the sustainable per-node multicast capacity in uniformly dense network by ad hoc routing is:

$$
\lambda=\Theta\left(\frac{W \cdot g(n) \eta(\sqrt{5} c)}{\frac{\sqrt{k} f^{3}(n)}{n}}\right)=\Theta\left(\frac{W}{\sqrt{k} f(n)}\right)
$$

\section{Multicast Capacity in Uniformly Dense Network by Cellular Routing}

In this section, we consider the impact of infrastructure in multicast capacity of a mobile network. Multicast flows will be routed through BSs. We divide the bandwidth in air channels into uplink bandwidth $W_{A}$ and downlink bandwidth $W_{C}$. We further assume that the bandwidth of optical fibers connecting $\mathrm{BSs}$ is $W_{B}$.

Definition 10: (Cellular Routing $R^{C}$ ) Cellular routing $R^{C}$ consists of three phases. In the first phase, a multicast source node routes the packets to a BS. In the second phase, the packets are routed to the cells that contain destinations. In the last phase, BSs of these cells broadcast packets to the destinations.

It is worth pointing out that such a routing cannot be established directly in mobile network. However, with the help of the mapping scheme presented in previous section, it is possible to generate a cellular multicast route in a random geometric graph.

\section{A. Upper Bounds in Uniformly Dense Network by Cellular Routing}

Since cellular routing $R^{C}$ is divided into three phases, the capacity of cellular routing $R^{C}$ is restricted by the worst case among three phases. We firstly explore the upper bound in each phase, then combine them together to obtain the overall upper bound.

1) Upper Bound in Phase 1: In phase 1, $n$ MSs act as sources, and try to forward their messages to one of the BSs. Scheduling policy $S^{*}$ is applied in this phase, and the uplink bandwidth for each BS is $W_{A}$.

Lemma 6: The per-node capacity upper bound of uniformly dense network with $m$ BSs and $n$ MSs, in phase 1 of cellular routing $R^{C}$, is :

$$
\lambda_{p 1}=O\left(\frac{m W_{A}}{n}\right)
$$


Proof: Because of the limit antennas, a BS can serve at most $\Theta(1)$ MSs in each time slot. That means one BS can at most receive $\Theta\left(W_{A}\right)$ traffic in each time slot. By applying a simple TDMA or FDMA scheme, the maximum of total available uplink resource is $\Theta\left(W_{A} \cdot m\right)$. All the $n$ MSs share this resource equally, which means that per-node capacity of each MS can not exceed $\Theta\left(\frac{m W_{A}}{n}\right)$.

2) Upper Bound in Phase 2: In phase 2, BSs exchange messages received from MSs through optical fibers, each of which has a bandwidth of $W_{B}$. We map the network only consisting BSs into a random geometric graph $G_{B}\left(m, W_{B}\right)$. $m$ vertices are used to represent the BSs. The capacities of edges connecting each pair of BSs $(i, j)$ are $W_{B}$. The next proposition holds in such graph.

Definition 11: (Inner and outer tessellation) Consider a torus, which is regularly divided into multiple squarelets. Assume that, in this torus, there is a subset $\Psi$ bounded by a closed and convex boundary $\mathcal{L}$. The inner tessellation of this torus, denoting as $\underline{\Psi}$, contains squarelets strictly within $\Psi$. And the outer tessellation is a union of $\underline{\Psi}$ and all the squarelets crossed by boundary $\mathcal{L}$, denoting as $\bar{\Psi}$.

Proposition 2: In a random geometric graph, traffic $\lambda$ is sustainable only if, for any partition $(S, D)$ of vertices, it holds:

$$
\lambda \sum_{s \in S} \sum_{d \in D} \leq \sum_{i \in S} \sum_{j \in D} \mu_{i j}^{n}
$$

Lemma 7: The per-node capacity upper bound of uniformly dense network with $m$ BSs and $n$ MSs, in phase 2 of cellular routing $R^{C}$, is :

$$
\lambda_{p 2}=O\left(\frac{m^{2} W_{B}}{k n}\right)
$$

Proof: From Proposition 2, we have a upper bound of per-node capacity:

$$
\lambda_{p 2} \leq \frac{\sum_{i: Y_{i}^{h} \in S_{\mathcal{L}}} \sum_{j: Y_{j}^{h} \in D_{\mathcal{L}}} \mu\left(Y_{i}^{h}, Y_{j}^{h}\right)}{\sum_{s: Y_{s}^{h} \in S_{\mathcal{L}}} \sum_{d: Y_{d}^{h} \in D_{\mathcal{L}}} \lambda_{s d}}
$$

where $\mathcal{L}$ is an arbitrary, simple, closed and convex curve, which divides $\mathcal{O}$ into $S_{\mathcal{L}}$ and $D_{\mathcal{L}}$.Define $\underline{S}_{\mathcal{L}}$ and $\bar{S}_{\mathcal{L}}$ as the inner and outer tessellation of $S_{\mathcal{L}}$, respectively. Define $\underline{D}_{\mathcal{L}}$ and $\bar{D}_{\mathcal{L}}$, similarly. We can carefully design above tessellations, such that the area of each squarelet in these tessellations is $(16+\delta) \gamma(n)$.

We first consider the numerator of (9). Denote $\mu_{B}^{m}=$ $\sum_{i: Y_{i}^{h} \in S_{\mathcal{L}}} \sum_{j: Y_{j}^{h} \in D_{\mathcal{L}}} \mu\left(Y_{i}^{h}, Y_{j}^{h}\right)$. Since $\forall i, j, \mu\left(Y_{i}^{h}, Y_{j}^{h}\right)=$ $W_{B}$, it is obvious that:

$$
\begin{aligned}
& \mu_{B}^{m} \geq W_{B} \cdot \sum_{Y_{i} \in \underline{S}_{\mathcal{L}}} \sum_{Y_{j} \in \underline{D}_{\mathcal{L}}} N_{b}\left(\underline{S}_{\mathcal{L}}\right) N_{b}\left(\underline{D}_{\mathcal{L}}\right) \\
& \mu_{B}^{m} \leq W_{B} \cdot \sum_{Y_{i} \in \bar{S}_{\mathcal{L}}} \sum_{Y_{j} \in \bar{D}_{\mathcal{L}}} N_{b}\left(\bar{S}_{\mathcal{L}}\right) N_{b}\left(\bar{D}_{\mathcal{L}}\right)
\end{aligned}
$$

Applying Lemma 1 , the following holds w.h.p.:

$$
\begin{aligned}
& \mu_{B}^{m} \geq \frac{1}{16} m^{2}(16+\delta)^{2} W_{B} \cdot \sum_{Y_{i} \in \underline{S}_{\mathcal{L}}} \sum_{Y_{j} \in \underline{D}_{\mathcal{L}}} \gamma^{2}(n) \\
& \mu_{B}^{m} \leq 16 m^{2}(16+\delta)^{2} W_{B} \cdot \sum_{Y_{i} \in \bar{S}_{\mathcal{L}}} \sum_{Y_{j} \in \bar{D}_{\mathcal{L}}} \gamma^{2}(n)
\end{aligned}
$$

Recall that $\gamma^{2}(n)=o(1)$ in uniformly dense network, (10) and (11) can be seen as upper and lower Riemann sum with mesh size decreasing to 0 as $n \rightarrow \infty$. As a result, we have:

$$
\mu_{B}^{m} \sim m^{2} W_{B} \int_{X \in S_{\mathcal{L}}} \int_{Y \in D_{\mathcal{L}}} d X d Y \sim m^{2} W_{B}
$$

Lemma 1 guarantee that number of source-destination pairs crossing $\mathcal{L}$ is $\Theta(k n)$ w.h.p., which is the interpretation of the denominator in (9). Combining with the result of numerator, it is evident that:

$$
\lambda_{p 2}=O\left(\frac{m^{2} W_{B}}{k n}\right)
$$

3) Upper Bound in Phase 3: In phase 3, each BS transmits messages in its own cell. Optimal scheduling policy $S *$ is applied in this phase again, and the downlink bandwidth for each BS is $W_{C}$. We map the network consisting of $m$ BSs and $n$ MSs into a random geometric graph $G_{C}\left(m+n, W_{C}\right)$. $m$ vertices are used to represent the BSs, and the other $n$ are for MSs. Edges only exist from BSs to MSs, with capacity $\mu\left(Y_{i}^{h}, X_{j}^{h}\right)$. There is no link among BSs, as well as MSs.

Proposition 3: Given an arbitrary non-increasing function s(x) with finite support and $X \in \mathcal{O}$, it holds:

$$
\int_{Y \in \mathcal{O}} s(f(n)\|Y-X\|) d Y \sim \frac{1}{f^{2}(n)}
$$

Lemma 8: The per-node capacity upper bound of uniformly dense network with $m$ BSs and $n$ MSs, in phase 3 of cellular routing $R^{C}$, is :

$$
\lambda_{p 3}=O\left(\frac{m W_{C}}{k n}\right)
$$

Proof: By Proposition 2, we can derive an expression of upper bound in phase 3 :

$$
\lambda_{p 3} \leq \frac{\sum_{i: Y_{i}^{h} \in U_{\mathcal{L}_{\mathcal{C}}}} \sum_{j: X_{j}^{h} \in V_{\mathcal{L}_{\mathcal{C}}}} \mu\left(Y_{i}^{h}, Y_{j}^{h}\right)}{\sum_{s: Y_{s}^{h} \in U_{\mathcal{L}_{\mathcal{C}}}} \sum_{d: Y_{d}^{h} \in V_{\mathcal{L}_{\mathcal{C}}}} \lambda_{s d}}
$$

where $\mathcal{L}_{\mathcal{C}}$ is an arbitrary, simple, closed and convex curve, which divides $\mathcal{O}$ into $U_{\mathcal{L}_{\mathcal{C}}}$ and $V_{\mathcal{L}_{\mathcal{C}}}$. Define $\underline{U}_{\mathcal{L}_{\mathcal{C}}}$ and $\bar{U}_{\mathcal{L}_{\mathcal{C}}}$ as the inner and outer tessellation of $U_{\mathcal{L}_{\mathcal{C}}}$, respectively. Define $\underline{V}_{\mathcal{L}_{\mathcal{C}}}$ and $\bar{V}_{\mathcal{L}_{\mathcal{C}}}$, similarly. We can carefully design above tessellations, such that the area of each squarelet in these tessellations is $(16+\beta) \gamma(n)$. Define $\bar{N}_{A_{U}}$ and $\underline{N}_{A_{U}}$ as the upper and lower bounds on the number of vertices within $\forall A_{U} \in U_{\mathcal{L}_{\mathcal{C}}}$. Define $\bar{N}_{A_{V}}$ and $\underline{N}_{A_{V}}$ for $V_{\mathcal{L}_{\mathcal{C}}}$ similarly. Define $\bar{d}_{A_{U}, A_{V}}$ and $\underline{d}_{A_{U}, A_{V}}$ as the maximal and minimal distance between points of $A_{U}$ and $A_{V}$. 
Denote $\mu_{C}^{m+n}=\sum_{i: Y_{i}^{h} \in U_{\mathcal{L}_{\mathcal{C}}}} \sum_{j: X_{j}^{h} \in V_{\mathcal{L}_{\mathcal{C}}}} \mu\left(Y_{i}^{h}, X_{j}^{h}\right)$. We can express the upper and lower bounds of $\mu_{C}^{m+n}$ as:

$$
\begin{aligned}
& \mu_{C}^{m+n} \geq \sum_{A_{U} \in \underline{U}_{\mathcal{L}_{\mathcal{C}}}} \sum_{A_{V} \in \underline{V}_{\mathcal{L}_{\mathcal{C}}}} \mu^{h}\left(\bar{d}_{A_{U}, A_{V}}\right) \underline{N}_{A_{U}} \underline{N}_{A_{V}} \\
& \mu_{C}^{m+n} \leq \sum_{A_{U} \in \bar{U}_{\mathcal{L}_{\mathcal{C}}}} \sum_{A_{V} \in \bar{V}_{\mathcal{L}_{\mathcal{C}}}} \mu^{h}\left(\underline{d}_{A_{U}, A_{V}}\right) \bar{N}_{A_{U}} \bar{N}_{A_{V}}
\end{aligned}
$$

We can select tessellations $\underline{U}_{\mathcal{L}_{\mathcal{C}}}, \bar{U}_{\mathcal{L}_{\mathcal{C}}}, \underline{V}_{\mathcal{L}_{\mathcal{C}}}$ and $\bar{V}_{\mathcal{L}_{\mathcal{C}}}$ carefully, such that their squarelets are of area $(16+\beta) \gamma(n)$. According to Lemma 1, we have:

$$
\begin{aligned}
\mu_{C}^{m+n} & \geq \frac{1}{16} m n(16+\beta)^{2} \sum_{A_{U} \in \underline{\underline{U}}_{\mathcal{L}_{\mathcal{C}}}} \sum_{A_{V} \in \underline{V}_{\mathcal{L}_{\mathcal{C}}}} \mu^{h}\left(\bar{d}_{A_{U}, A_{V}}\right) \gamma^{2}(n) \\
\mu_{C}^{m+n} & \leq 16 m n(16+\beta)^{2} \sum_{A_{U} \in \bar{U}_{\mathcal{L}_{\mathcal{C}}}} \sum_{A_{V} \in \bar{V}_{\mathcal{L}_{\mathcal{C}}}} \mu^{h}\left(\underline{d}_{A_{U}, A_{V}}\right) \gamma^{2}(n)
\end{aligned}
$$

Since $\gamma^{2}(n)=o(1)$ in uniformly dense network, (13) and (14) can be seen as upper and lower Riemann sum with mesh size decreasing to 0 as $n \rightarrow \infty$. Combining with Proposition 3 and Corollary 1, we have:

$$
\begin{aligned}
\mu_{C}^{m+n} & \sim \int_{X \in V_{\mathcal{L}_{\mathcal{C}}}} \int_{Y \in U_{\mathcal{L}_{\mathcal{C}}}} m n \mu\left(d_{Y X}\right) d Y d X \\
& \sim m n W_{C} \cdot \frac{f^{2}(n)}{n} \int_{X \in V_{\mathcal{L}_{\mathcal{C}}}} \int_{Y \in U_{\mathcal{L}_{\mathcal{C}}}} s(f(n)\|Y-X\|) d Y d X \\
& \sim m W_{C} \cdot f^{2}(n) \cdot \frac{1}{f^{2}(n)} \int_{X \in V_{\mathcal{L}_{\mathcal{C}}}} d X \\
& \sim m W_{C}
\end{aligned}
$$

Meanwhile, applying Lemma 1 , it is easy to prove that number of source-destination pairs crossing boundary $\mathcal{L}$ in phase 3 is $\Theta(k n)$, w.h.p.. It is an interpretation of denominator in (12). Now we can conclude that an multicast capacity upper bound in phase 3 of cellular routing $R^{C}$ is:

$$
\lambda_{p 3}=O\left(\frac{m W_{C}}{k n}\right)
$$

As previously described, cellular routing $R^{C}$ is a serial connection of the above 3 phases. According to its characteristic, the overall capacity can not exceed the capacity of any element in the serial connection. With Lemma 6-8, we have the following theorem.

Theorem 3: The upper bound of per-node multicast capacity in uniformly dense network by cellular routing $R^{C}$ is:

$$
O\left(\min \left[\frac{m W_{A}}{n}, \frac{m^{2} W_{B}}{k n}, \frac{m W_{C}}{k n}\right]\right)
$$

\section{B. Lower Bounds in Uniformly Dense Network by cellular} routing $R^{C}$

Similar to the derivation of upper bounds by cellular routing $R^{C}$, we derive lower bounds of cellular routing $R^{C}$ in 3 phase, respectively. Then a combination of the lower bounds is presented.

\section{1) Lower Bound in Phase 1:}

Lemma 9: A traffic rate $\Theta\left(\frac{m W_{A}}{n}\right)$ is sustainable from any MS to infrastructure system in phase 1 of cellular routing $R^{C}$.

Proof: Since Lemma 1 provides lower and upper bounds for number of home-points in a certain area, we can easily obtain the following assertion by applying it: the maximal number of home-points within each cell(or the coverage area of each BS) is $\Theta\left(\frac{n}{m}\right)$ w.h.p.. As is proved in Section III-B, a transmission range of $R_{T}=\Theta\left(\frac{1}{\sqrt{n}}\right)$ causes no interference between adjacent BSs. Therefore, the uplink bandwidth $W_{A}$ is fully available in each cell, and is share by at most $\Theta\left(\frac{n}{m}\right)$ MSs. At a result, a simple FDMA scheme can be employed to achieved throughput of:

$$
\lambda_{p 1} \geq \Theta\left(\frac{W_{A}}{n / m}\right)=\Theta\left(\frac{m W_{A}}{n}\right)
$$

\section{2) Lower Bound in Phase 2:}

Lemma 10: A traffic rate $\Theta\left(\frac{m^{2} W_{B}}{k n}\right)$ is sustainable between BSs in phase 2 of cellular routing $R^{C}$.

Proof: Consider the random geometric graph mapping of BSs described in Section IV-A, the maximum traffic from source squarelet $S$ to destination squarelet $D$ is upper-bounded by $\lambda \cdot k n$. This traffic is sustainable, only if none of the edges in the route is overload. And it holds w.h.p., if:

$$
\frac{\lambda_{p 2} \cdot k n}{N_{b}(S) N_{b}(D)} \sim \frac{\lambda \cdot k n}{m^{2}} \leq W_{B}
$$

Therefore, $\forall \lambda_{p 2} \leq \Theta\left(\frac{m^{2} W_{B}}{k n}\right)$ is sustainable in phase 2 of cellular routing $R^{C}$.

3) Lower Bound in Phase 3: Let $f_{i}$ denote the number of multicast flows, each of which has as least one destination inside $i$ th BS's cell(or transmission range). We borrow the next lemma from [10] to facilitate our proof.

Lemma 11: When $n_{s}>\max \left(\frac{32 m \log m}{k} \log \frac{52 m}{k}, \frac{16 m}{k} \log (2 n)\right)$ is satisfied, the variable $\max _{j}^{m} f_{j}$ is $\Theta\left(\frac{n_{s} k}{m}\right)$ w.h.p., where $n_{s}$ is the number of source nodes.

Lemma 12: A traffic rate $\Theta\left(\frac{m W_{C}}{k n}\right)$ is sustainable from one BS to MSs in phase 3 of cellular routing $R^{C}$.

Proof: Recall the assumption of our model that all the $n$ MSs in the network act as source node of a multicast flow. A direct corollary of Lemma 11 in our model is: for an arbitrary cell, there are at most $\Theta\left(\frac{n k}{m}\right)$ destinations that it needs to serve. As the BSs do not interfere with each other, a downlink bandwidth of $W_{C}$ is available in each cell. A FDMA scheme that divides the frequency into $\Theta\left(\frac{n k}{m}\right)$ parts can provide a pernode throughput as followed:

$$
\lambda_{p 3}=\Theta\left(\frac{W_{C}}{(k n / m)}\right)=\Theta\left(\frac{m W_{C}}{k n}\right)
$$

An overall lower bound of cellular routing $R^{C}$ can be obtained by combining the lower bounds in 3 phases. With Lemma $9,10,12$, we can draw the following conclusion: 
Theorem 4: The lower bound of per-node multicast capacity in uniformly dense network by cellular routing $R^{C}$ is:

$$
\Theta\left(\min \left[\frac{m W_{A}}{n}, \frac{m^{2} W_{B}}{k n}, \frac{m W_{C}}{k n}\right]\right)
$$

\section{Multicast Capacity in Uniformly Dense NETWORK BY HYBRID ROUTING}

At the beginning of this section, we analyze multicast capacity upper bound for arbitrary hybrid routing. Then matching lower bound and corresponding routing scheme are presented. The hybrid routing utilizes both ad hoc routing and cellular routing, with the purpose of further improving the network capacity and system throughput. It is worth noticing that optimal hybrid routings allows packets to access the infrastructure system at most once. A concise explanation is that accessing infrastructure several times will directly decrease the efficiency of frequency and time resources, especially the uplink/downlink bandwidth.

\section{A. Upper and Lower Bounds by Hybrid Routing}

Theorem 5: In uniformly dense network, the upper bound of per-node multicast capacity by any hybrid routing is:

$$
O\left\{\max \left[\frac{W}{\sqrt{k} f(n)}, \min \left(\frac{m W_{A}}{n}, \frac{m^{2} W_{B}}{k n}, \frac{m W_{C}}{k n}\right)\right]\right\}
$$

Proof: Combining Theorem 1 and 3 , it is obvious that, in uniformly dense network, per-node multicast capacity $\lambda$ is upper-bounded by:

$$
\lambda=O\left(\frac{W}{\sqrt{k} f(n)}\right)+O\left[\min \left(\frac{m W_{A}}{n}, \frac{m^{2} W_{B}}{k n}, \frac{m W_{C}}{k n}\right)\right]
$$

By the limit theory, we can rewrite the above expression as:

$$
\lambda=O\left\{\max \left[\frac{W}{\sqrt{k} f(n)}, \min \left(\frac{m W_{A}}{n}, \frac{m^{2} W_{B}}{k n}, \frac{m W_{C}}{k n}\right)\right]\right\}
$$

Definition 12: (Hybrid Routing $R^{H}$ ) Hybrid Routing $R^{H}$ evaluates both pure ad hoc routing and cellular routing $R^{C}$, and adaptively selects a better scheme, which provides larger throughput, to route the packets.

Applying Theorem 2 and 4 together, the following theorem holds beyond doubt.

Theorem 6: By hybrid routing $R^{H}$, the sustainable pernode multicast throughput in uniformly dense network is:

$$
\Theta\left\{\max \left[\frac{W}{\sqrt{k} f(n)}, \min \left(\frac{m W_{A}}{n}, \frac{m^{2} W_{B}}{k n}, \frac{m W_{C}}{k n}\right)\right]\right\}
$$

Theorem 6 shows that our hybrid routing $R^{H}$ is optimal in order sense. Moreover, it proves that the upper bound purposed by Theorem 5 is tight.
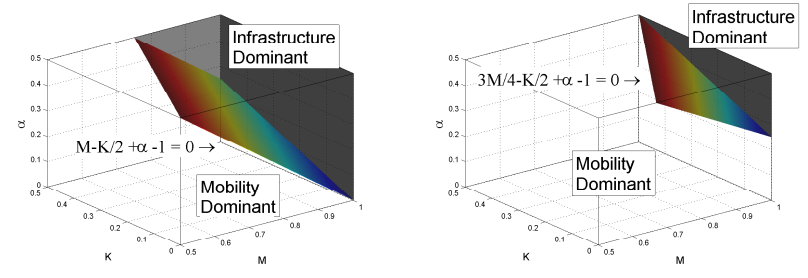

Fig. 2. Graphical illustration of capacity regimes in uniformly dense network by hybrid routing. The per-node capacity is a function of $m=\Theta\left(n^{M}\right), k=$ $\Theta\left(n^{K}\right), W_{B}=\Theta\left(W \cdot n^{\varphi}\right)$ and $f(n)=n^{\alpha}$. The left one accounts for $\varphi>-M$, where wireless access to infrastructure is the bottleneck; the right one stands for the situation that infrastructure slow downs the transmission rate(Here we take $\varphi=\frac{-5 M}{4}$ for example).

\section{B. Discussion on Capacity Regimes}

Proposition 4: The optimal frequency allocation between $W_{A}$ and $W_{C}$ is:

$$
W_{A}=\frac{W}{k+1}, \quad W_{C}=\frac{k W}{k+1}
$$

Proof: The optimal frequency allocation focuses on maximizing the uplink and downlink capacity between MSs and BSs. According to Theorem 6 , the capacity $\lambda_{U D}$ that can be provided by uplink and downlink transmissions is $\lambda_{U D}=$ $\Theta\left[\min \left(\frac{m W_{A}}{n}, \frac{m W_{C}}{k n}\right)\right]$. If $\frac{m W_{A}}{n}>\frac{m W_{C}}{k n}$, we have

$$
\lambda_{U D}=\frac{m W_{C}}{k n}<\frac{m W}{(k+1) n}
$$

If $\frac{m W_{A}}{n}<\frac{m W_{C}}{k n}$, we have

$$
\lambda_{U D}=\frac{m W_{A}}{n}<\frac{m W}{(k+1) n}
$$

However, if we allocate $W$ as $W_{A}=\frac{W}{k+1}, W_{C}=\frac{k W}{k+1}$, it results:

$$
\lambda_{U D}=\frac{m W}{(k+1) n}
$$

The multicast capacity in uniformly dense network by hybrid routing can be represented by the following parameters: $m=\Theta\left(n^{M}\right), k=\Theta\left(n^{K}\right), W_{B}=\Theta\left(W \cdot n^{\varphi}\right)$ and $f(n)=n^{\alpha}$. If $\varphi>-M$, the bottleneck of cellular routing falls in the wireless access. Otherwise, the backbone transmission becomes the limitation. Fig. 2 provides graphical illustration of capacity regimes.

\section{Multicast Capacity in Non-Uniformly Dense NETWORK}

In the previous sections, we discuss the multicast capacity of mobile uniformly dense network, where $f(n) \sqrt{\gamma(n)}=o(1)$. Now we will present the results in mobile non-uniformly dense network. As is described, mobility in non-uniformly dense network can be further divided into two regimes: weak and trivial. Mobility only helps in intra-cluster message delivering in weak mobility regime. As a result, we present a mix routing scheme for both intra-cluster and inter-cluster multicast transmission. Meanwhile, we point out that in trivial mobility 


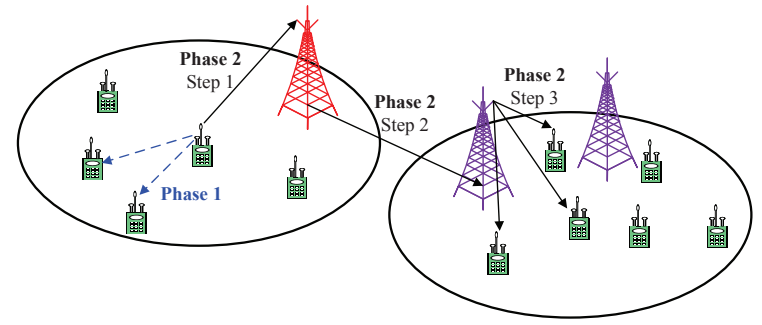

Fig. 3. An example of hybrid routing $\widetilde{R}$ in weak mobility regime

regime, the impact of mobility can be ignored. Therefore, capacity results in previous literature can be applied in this regime.

In non-uniformly dense network without the support of BSs, a larger transmission range should be adopted to guarantee connectivity. In [3], it is proved that this transmission range should be $R_{T}=\Omega(1 / \sqrt{n})$, which only provides a capacity of $\Theta\left(\frac{n_{c}}{n^{2} \log n_{c}}\right)$. The poor capacity is a consequence of more interference brought by larger transmission range. Considering this, we propose to decrease the transmission range of nodes, and employ BSs to guarantee connectivity. A useful lemma is cited from [11], so as to facilitate our proof.

Lemma 13: Nodes with transmission range $R_{T}=r \sqrt{\frac{n_{c}}{n}}$ will cause no interference to those in other clusters, with high probability.

According to the new transmission range, we propose a new scheduling scheme.

Definition 13: (Scheduling Scheme $\widetilde{S}$ ) $\widetilde{S}$ schedules node $i$ transmit to node $j$ at time slot $t$, only if the following two condition are both satisfied: 1) $\left\|Z_{i}(t)-Z_{j}(t)\right\|<R_{T}=$ $c_{T} \cdot r \sqrt{\frac{n_{c}}{n}}$, and 2) For any other node $l$, whether active or silent, $\left\|Z_{l}(t)-Z_{j}(t)\right\|>(1+\Delta) R_{T}$.

\section{A. Multicast Capacity in Weak Mobility Regime}

Under scheduling scheme $\widetilde{S}$, MSs in different clusters cannot communicate with each other directly in air channels. A hybrid routing is proposed to finish the transmission.

Definition 14: (Hybrid Routing $\widetilde{R}$ ) Hybrid Routing $\widetilde{R}$ consists of 2 phases. In phase 1 , each source node transmits packets to destinations in its own cluster. In phase 2, each source node employs cellular routing (as defined in Definition 10) to transmit packets to destinations in other clusters.

Fig. 2 gives an example of hybrid routing $\widetilde{S}$ in weak mobility regime.

1) Multicast Capacity in Phase 1 of $\widetilde{R}$ : Since there is no inter-cluster interference in phase 1 , each cluster acts like a sub-network independently. Next, we will prove that these subnetworks are uniformly dense in weak mobility regime, and extend the results in uniformly dense network into this phase.

Theorem 7: In weak mobility regime, each cluster forms an independent sub-network, which is uniformly dense.

Proof: By Lemma 13, it is obvious that ad hoc transmissions within one cluster are independent of those in other clusters, since the transmission range is less than the interference range.

It is crucial to determine the key parameters $\left(\tilde{f}(n), \tilde{n}, \tilde{m}, \tilde{k}, \tilde{n}_{c}, \tilde{R}_{T}\right)$ of an arbitrary sub-network, if we want to prove it to be uniformly dense. A direct application of Chernoff bound results in the following expressions. For $\forall \varepsilon>0$, these hold w.h.p.:

$$
\begin{aligned}
& \frac{(1-\varepsilon) n}{n_{c}} \quad<\tilde{n}<\frac{(1+\varepsilon) n}{n_{c}} \\
& \frac{(1-\varepsilon) m}{n_{c}} \quad<\tilde{m}<\frac{(1+\varepsilon) m}{n_{c}} \\
& \frac{(1-\varepsilon) k}{n_{c}} \quad<\tilde{k}<\frac{(1+\varepsilon) k}{n_{c}}
\end{aligned}
$$

The definition of $\widetilde{S}$ already provides that $\widetilde{R}_{T}=$ $\left(c_{T} \cdot r \sqrt{\frac{n_{c}}{n}}\right) / r=c_{T} \sqrt{\frac{n_{c}}{n}}$. Also, by the definition of cluster model, home-points in each cluster are randomly, independently and uniformly placed, so we have $\tilde{n}_{c}=\tilde{n}$. Since that each cluster has a radius of $r=\Theta\left(n^{-R}\right)$, the radius of each sub-network is $r+D / f(n)$, where $\mathrm{D}$ is a constant such that $D=\sup \{d \mid s(d)>0\}$.

Now we can normalize the area of each sub-network into 1. Correspondingly, we have $\tilde{f}(n)=\frac{1}{r+D / f(n)} \sim n^{R}$. According to the definition of weak mobility, we have $f(n) \sqrt{\widetilde{\gamma}(n)}=$ $o(1)$, which can be extended as:

$$
\begin{aligned}
f(n) \sqrt{\tilde{\gamma}(n)} & =f(n) \cdot \frac{1}{r} \sqrt{\frac{\log \left(n / n_{c}\right)}{n / n_{c}}} \\
& \sim \tilde{f}(n) \sqrt{\frac{\log \tilde{n}}{\tilde{n}}}=\tilde{f}(n) \sqrt{\frac{\log \tilde{n}_{c}}{\tilde{n_{c}}}}
\end{aligned}
$$

It yields:

$$
\tilde{f}(n) \sqrt{\frac{\log \tilde{n}_{c}}{\tilde{n}_{c}}}=o(1)
$$

Applying Lemma 2 in all sub-networks guarantees that each of them is uniformly dense.

With Theorem 7, we can map our results in Section III directly into phase 1 of $\widetilde{R}$, obtain the following theorem.

Theorem 8: The per-node multicast capacity of phase 1 in $\widetilde{R}$ is

$$
\widetilde{\lambda}_{p 1}=\Theta\left(\frac{W \sqrt{n_{c}}}{n^{R} \sqrt{k}}\right)
$$

2) Multicast Capacity in Phase 2 of $\widetilde{R}$ : Since we apply cellular routing $R^{C}$ in phase 2 hybrid routing $\widetilde{R}$, we can further divide phase 2 of $\widetilde{R}$ into three serial steps. In the first step, source nodes deliver their packets to BSs in the same cluster, through wireless channels with uplink bandwidth $W_{A}$. Then, with the help of infrastructure, each packet is forwarded to the clusters, where the destinations nodes locate. The traffic rate between $\mathrm{BSs}$ is $W_{B}$. In the last step, BSs in each cluster transmit the messages to the destinations in wireless channels with downlink bandwidth $W_{C}$. If we can determine the parameters in phase 2 of $\widetilde{R}$ similar to those in cellular routing $R^{C}$, previous results can be mapped into this phase of $\widetilde{R}$. 
In the first step, transmissions in different clusters cause no interference to each other, which is guaranteed by Theorem 7. Considering a sub-network formed by an arbitrary cluster, there are $\tilde{n}=\Theta\left(n / n_{c}\right)$ source nodes try to transmit their packets to $\tilde{m}=\Theta\left(m / n_{c}\right)$ BSs. Consequently, a direct mapping of Lemma 6 and 9 ensures the following assertion:

Lemma 14: The per-node capacity in the first step of $\widetilde{R}$ 's phase 2 is:

$$
\Theta\left(\frac{\tilde{m} W_{A}}{\tilde{n}}\right)=\Theta\left(\frac{m W_{A}}{n}\right)
$$

In the second step, exchanges of information take place in the infrastructure system. The transmissions involve $m$ BSs and $n$ MSs, which is the same as phase 2 in $R^{C}$. The only difference is the number of destinations nodes in other clusters, which is $k-\tilde{k}$ in this step. Therefore, Lemma 7 and 10 can be applied in this step with the above parameters, and result in the next lemma:

Lemma 15: The per-node capacity in the second step of $\widetilde{R}$ 's phase 2 is:

$$
\Theta\left(\frac{m^{2} W_{B}}{(k-\tilde{k}) n}\right)=\Theta\left(\frac{n_{c} m^{2} W_{B}}{\left(n_{c}-1\right) n k}\right)
$$

In the last step, BSs transmit packets to all the destinations in the same cluster. As previously described, there are $\tilde{m}$ BSs and $\tilde{n}$ MSs in each cluster. Number of destination nodes in each cluster is $k^{(d e s)}=\frac{(k-\tilde{k})}{n_{c}}=\Theta\left(\frac{k}{n_{c}}-\frac{k}{n_{c}^{2}}\right)=\Theta\left(\frac{k}{n_{c}}\right)$, which can be proved by utilizing Lemma 1 . Substituting the above parameters into Lemma 8 and 12, the following lemma holds in this step.

Lemma 16: The per-node capacity in the last step of $\widetilde{R}$ 's phase 2 is:

$$
\Theta\left(\frac{\tilde{m} W_{C}}{k^{(d e s)} \tilde{n}}\right)=\Theta\left(\frac{m n_{c} W_{C}}{k n}\right)
$$

Now that we have the results in all the serial components of hybrid routing $\widetilde{R}$, the overall capacity should be restricted by the minimum throughput of all the components. As a result, we provide the following theorem, which holds w.h.p. in weak mobility regime.

Theorem 9: In weak mobility regime, the per-node multicast capacity of mobile ad hoc network with infrastructure support, by hybrid routing $\widetilde{R}$, is:

$$
\Theta\left[\min \left(\frac{W \sqrt{n_{c}}}{n^{R} \sqrt{k}}, \frac{m W_{A}}{n}, \frac{n_{c} m^{2} W_{B}}{\left(n_{c}-1\right) n k}, \frac{m n_{c} W_{C}}{n k}\right)\right]
$$

\section{B. Multicast Capacity in Trivial Mobility Regime}

Applying the connectivity criterion in [17], the critical transmission range within each cluster is $R_{T}^{\prime}=r \sqrt{\log (\tilde{n}) / \pi \tilde{n}}$ when $f(n) \sqrt{\tilde{\gamma}(n)}=\omega(1)$. From Lemma 13, it is obvious that $R_{T}^{\prime}$ will cause no inter-cluster interference. We can map each cluster into sub-network almost the same as Theorem 7. The only difference is that $\widetilde{R}_{T}=\sqrt{\log (\tilde{n}) / \pi \tilde{n}}$.

Theorem 10: In trivial mobility regime, nodes' mobility are negligible and the whole network acts as a static one.
Proof: Since the clusters are independent with each other in trivial mobility regime, we only need to prove that each node acts like a static one within each cluster. It is equivalent to prove that the following four conditions hold for any time slots $t_{0}$ and $\left.t\left(t_{0}<t\right): 1\right)$ Any pair $i, j$ that can communicate at $t_{0}$ will be able to communicate at $\left.t ; 2\right)$ any pair $i, j$ that cannot communicates at $t_{0}$ still cannot communicate at $t ; 3$ ) Any pair $i, j$ that cause interference to each other at $t_{0}$ will still cause interference to each other at $t$; 4)Any pair $i, j$ that cause no interference to each other at $t_{0}$ will not cause any interference to each other at $t$.

According to the mobility model, a node can at most move $D / \widetilde{f}(n)$ away from its home-point. Hence the maximal change of distance between two nodes is $4 D / \widetilde{f}(n)$. Considering the definition of trivial mobility regime, it holds that $\widetilde{R}_{T}=\omega\left(\frac{1}{\tilde{f}(n)}\right)$. We first consider the probability of condition $1)$ :

$$
\begin{aligned}
P_{1} & =\operatorname{Pr}\left\{d_{i j}(t) \leq \widetilde{R}_{T} \mid d_{i j}\left(t_{0}\right) \leq \widetilde{R}_{T}\right\} \\
& =\operatorname{Pr}\left\{d_{i j}\left(t_{0}\right) \leq \widetilde{R}_{T}-\frac{4 D}{f(n)} \mid d_{i j}\left(t_{0}\right) \leq \widetilde{R}_{T}\right\} \\
& =\frac{\pi\left(\widetilde{R}_{T}-\frac{4 D}{f(n)}\right)^{2}}{\pi \widetilde{R}_{T}^{2}} \rightarrow 1
\end{aligned}
$$

The probability of condition 2) follows:

$$
\begin{aligned}
P_{2} & =1-\operatorname{Pr}\left\{d_{i j}(t) \leq \widetilde{R}_{T} \mid d_{i j}\left(t_{0}\right)>\widetilde{R}_{T}\right\} \\
& =1-\operatorname{Pr}\left\{d_{i j}\left(t_{0}\right) \leq \frac{4 D}{\widetilde{f}(n)} \mid d_{i j}\left(t_{0}\right)>\widetilde{R}_{T}\right\} \\
& =1-\frac{\operatorname{Pr}\left\{\widetilde{R}_{T}<d_{i j}\left(t_{0}\right) \leq \frac{4 D}{\widetilde{f}(n)}\right\}}{\operatorname{Pr}\left\{d_{i j}\left(t_{0}\right) \leq \widetilde{R}_{T}\right\}} \\
& \rightarrow 1-\frac{0}{\operatorname{Pr}\left\{d_{i j}\left(t_{0}\right) \leq \widetilde{R}_{T}\right\}}=1
\end{aligned}
$$

Let $A_{3}$ denotes the event of $\widetilde{R}_{T} \leq d_{i j}\left(t_{0}\right) \leq(1+\Delta) \widetilde{R}_{T}$, then the probability of condition 3 ) can be expressed as:

$$
\begin{aligned}
P_{3} & =\operatorname{Pr}\left\{\widetilde{R}_{T} \leq d_{i j}(t) \leq(1+\Delta) \widetilde{R}_{T} \mid A_{3}\right\} \\
& =\operatorname{Pr}\left\{\widetilde{R}_{T}+\frac{4 D}{f(n)} \leq d_{i j}\left(t_{0}\right) \leq(1+\Delta) \widetilde{R}_{T}-\frac{4 D}{f(n)} \mid A_{3}\right\} \\
& =\frac{\pi\left\{\left[(1+\Delta) \widetilde{R}_{T}-\frac{4 D}{f(n)}\right]-\left(\widetilde{R}_{T}+\frac{4 D}{f(n)}\right)\right\}^{2}}{\pi\left[(1+\Delta) \widetilde{R}_{T}-\widetilde{R}_{T}\right]^{2}} \\
& =\frac{\left[\Delta \widetilde{R}_{T}-\frac{8 D}{f(n)}\right]^{2}}{\left[\Delta \widetilde{R}_{T}\right]^{2}} \rightarrow 1
\end{aligned}
$$

Derivation of condition 4)'s probability is similar to that of condition 2). Therefore, we skip the detail and present the result: $P_{4} \rightarrow 1$ when $n \rightarrow \infty$.

To sum up, all of the 4 conditions hold for any time slots $t_{0}$ and $t\left(t_{0}<t\right)$ w.h.p., which means that communication states for all the nodes remain the same. As a result, mobility is 
negligible because it causes no change in the network status.

With the help of Theorem 8, we can extend existing results of static network into trivial mobility regime of mobile network. Since pervious works, such as [4], [9], [10], [11], etc., have already discussed capacity of static network with infrastructure support, one can refer to theses works for more details.

\section{CONCLUSION}

This paper analyzes the multicast capacity in mobile ad hoc network with infrastructure support. Node distribution follows a cluster model, which characterizes the spatial inhomogeneity of realistic mobility. On one hand, mobility is classified into three regimes: strong, weak and trivial. It is showed that, only in the first regime can mobility help to forward the intercluster multicast transmission. When it is in weak regime, mobility is only helpful in delivering inter-cluster packets. Moreover, mobility's impact is negligible when it falls into trivial regime. On the other hand, infrastructure support can essentially improve the network capacity. Especially in the last two regimes, BSs play a major role in forwarding inter-cluster packets. Hybrid routing schemes are proposed to achieve matching upper and lower bounds in each of the regimes. It is worth pointing out that our work generalizes results in previous works on hybrid network, impact of mobility and multicast transmissions, as well as any combinations of the above. Our results are instructive in the design of real hybrid system combining cellular and ad hoc networks.

\section{REFERENCES}

[1] P. Gupta and P. R. Kumar, "The capacity of wireless networks," IEEE Trans. Inf. Theory, vol. 46, no. 2, pp. 388-404, 2000.

[2] M. Grossglauser and D. N. C. Tse, "Mobility increases the capacity of ad hoc wireless networks," IEEE/ACM Trans. Networking, vol. 10, no. 4, pp. $477-486,2002$.

[3] M. Garetto, P. Giaccone, and E. Leonardi, "Capacity scaling in delay tolerant networks with heterogeneous mobile nodes," in ACM MobiHoc 2007, New York, USA, 2007, pp. 41-50.

[4] B. Liu, Z. Liu, and D. Towsley, "On the capacity of hybrid wireless networks," in IEEE Infocom 2003, vol. 2, San Francisco, USA, 2003, pp. $1543-1552$.

[5] U. C. Kozat and L. Tassiulas, "Throughput capacity of random ad hoc networks with infrastructure support," in ACM MobiCom 2003, New York, USA, 2003, pp. 55-65.

[6] A. Agarwal and P. R. Kumar, "Capacity bounds for ad hoc and hybrid wireless networks," SIGCOMM Comput. Commun. Rev., vol. 34, no. 3, pp. 71-81, 2004.

[7] X.-Y. Li, "Multicast capacity of wireless ad hoc networks," IEEE/ACM Trans. Networking, vol. 17, no. 3, pp. 950-961, 2009.

[8] X.-Y. Li, Y. Liu, S. Li, and S. Tang, "Multicast capacity of wireless ad hoc networks under gaussian channel model," IEEE/ACM Trans. Networking, vol. 18, no. 4, pp. 1145-1157, 2010.

[9] X. Mao, X.-Y. Li, and S. Tang, "Multicast capacity for hybrid wireless networks," in ACM MobiHoc 2008, Hong Kong, China, 2008, pp. 189198.

[10] X.-Y. Li, X. Mao, and S. Tang, "Closing the gap of multicast capacity for hybrid wireless networks," 2009, [Online]. Available: http://www.cs.iit.edu/ xli.

[11] W. Huang, X. Wang, and Q. Zhang, "Capacity scaling in mobile wireless ad hoc network with infrastructure support," in IEEE ICDCS, 2010, Genoa, Italy, 2010, pp. 848-857.

[12] M. J. Neely and E. Modiano, "Capacity and delay tradeoff for ad hoc mobile networks," IEEE Trans. Inf. Theory, vol. 51, no. 6, pp. 1917 1937, 2005.
[13] J. H. Kang, W. Welbourne, B. Stewart, and G. Borriello, "Extracting places from traces of locations," SIGMOBILE Mob. Comput. Commun. Rev., vol. 9, no. 3, pp. 58-68, 2005.

[14] G. Sharma, R. Mazumdar, and N. B. Shroff, "Delay and capacity tradeoffs in mobile ad hoc networks: A global perspective," IEEE/ACM Trans. Networking, vol. 15, no. 5, pp. 981-992, 2007.

[15] X. Lin, G. Sharma, R. R. Mazumdar, and N. B. Shroff, "Degenerate delay-capacity tradeoffs in ad-hoc networks with brownian mobility," IEEE/ACM Trans. Networking, vol. 14, no. SI, pp. 2777-2784, 2006.

[16] M. Garetto, P. Giaccone, and E. Leonardi, "Capacity scaling in ad hoc networks with heterogeneous mobile nodes: The super-critical regime," IEEE/ACM Trans. Networking, vol. 17, no. 5, pp. 1522-1535, 2009.

[17] P. Gupta and P. R. Kumar, "Critical power for asymptotic connectivity," in IEEE Conference on Decision and Control, 1998, vol. 1, Florida, USA, 1998, pp. 1106-1110. 